\title{
CONTRIBUIÇÃO AO ESTUDO ALELOPÁTICO DE ÁGUA RECUPERADA DE [Chamomilla recutita (L.) RAUSCHERT]
}

\section{CONTRIBUTION TO THE ALLELOPATHIC STUDY OF RECOUPED WATER FROM [Chamomilla recutita (L.) RAUSCHERT]}

SANTOS, Luciana M. dos $^{1}$, SENS, Rafaele C. V. ${ }^{1}$,
DIAS, Josiane F.G. ${ }^{1}$, BORSATO, Aurélio V. ${ }_{1^{*}}$, Miguel, Obdulio G. ${ }^{2}$, MIGUEL, Marilis D.

${ }^{1}$ Laboratório de Farmacotécnica, Departamento de Farmácia, Universidade Federal do Paraná, 80210-170, Curitiba-PR, Brasil.

${ }^{2}$ Laboratório de Fitoquímica, Departamento de Farmácia, Universidade Federal do Paraná, 80210-170, Curitiba-PR, Brasil.

${ }^{3}$ Eng.-. Agr $_{-}^{\circ}$, Pesquisador A, Embrapa Pantanal, 79320-900, Corumbá-MS, Brasil. *e-mail: dallarmi@ufpr.br

\section{RESUMO:}

Este trabalho buscou analisar a influência alelopática de águas recuperadas de [Chamomilla recutita (L.) Rauschert] sobre o crescimento e germinação de sementes de Lactuca sativa. Os resultados demonstraram que apesar da composição das águas recuperadas após 3 horas, 6 horas e 9 horas de secagem a $70^{\circ} \mathrm{C}$ ser diferente, esta variação não se mostrou representativa nos resultados alelopáticos. Em todos os tempos de secagem, a água recuperada influenciou o crescimento do hipocótilo e a germinação das sementes de Lactuca sativa.

Palavras-chave: Alelopatia, Lactuca sativa, Chamomilla recutita.

\section{ABSTRACT:}

This study aimed to analyzed the allelophatic influence from recouped water of [Chamomilla recutita (L.) Rauschert] on growth and germination of seeds of Lactuca sativa. The results had demonstrated that in spite of the composition of recouped waters after 3 hours, 6 hours and 9 hours of drying at $70^{\circ} \mathrm{C}$ is different, this variation didn't show representative in the allelophatic results. In all of the times of drying, the recouped water influenced the growth of hypocotyls and germination of seeds from Lactuca sativa.

Key words: Allelopathy, Lactuca sativa, Chamomilla recutita.

\section{INTRODUÇÃO}

A camomila (Matricaria chamomilla L.) é uma planta da família Asteraceae que teve sua nomenclatura modificada para [Chamomilla recutita (L.) Rauschert] em 1989 (Tucker; Duke; Foster, 1989). Espécie herbácea anual conhecida popularmente como 
camomila, camomila alemã, camomila comum, camomila vulgar, camomila-dosalemães, camomilinha, maçanilha, macela, macela-nobre, marcela galega e matricaria (CORRÊA JUNIOR, 1994; FARMACOPÉIA, 1996; DALLA COSTA E MIGUEL, 2001; LORENZI \& MATOS, 2002).

No Paraná, cerca de 3 mil hectares cultivados com plantas medicinais, 1800 hectares correspondem a camomila resultando numa produção de 600 toneladas. A zona produtora de camomila abrange principalmente os municípios de Mandirituba, São José dos Pinhais e Campo Largo, que são responsáveis por mais de $85 \%$ da produção estadual (DALLA COSTA, 2001; CORRÊA JUNIOR \& ROSSI, 2007; OLIVEIRA, 2009).

É uma das plantas mais utilizadas pela medicina tradicional européia, apresentando diversas ações, entre elas: emenagoga, sedativa, estimulante do apetite, auxílio na cicatrização da pele, anti-séptica, alívio de inflamação das gengivas (LORENZI \& MATOS, 2002), antiespasmódica, antiflogística, carminativa, tônica, emoliente, refrescante e antiinflamatória (TESKE \& TRENTINI, 1995). A atividade predominante do extrato aquoso é a espasmolítica, enquanto que no extrato alcoólico é a antiflogística (TESKE \& TRENTINI, 1995).

Cerca de 120 constituintes químicos foram identificados na camomila como metabólitos secundários, incluindo 28 terpenóides, 36 flavonóides e 52 compostos adicionais com potencial atividade farmacológica. O bisabolol e o camazuleno, que fazem parte do óleo essencial apresentam propriedades calmantes e antiinflamatórias, tendo aplicabilidade no tratamento de cólica gástrica, gastrites, gengivites, faringites e laringites, inflamações do trato respiratório, do útero, reto e hemorróidas. Flavonóides como a apigenina e a lutelina também exibem atividade antiinflamatória (SALAMON, 1992). COSTA (1987) cita a presença de fitosterol, açúcares, taninos, colina, vitaminas $\mathrm{C}$ e $\mathrm{B}$, inositol, cumarinas, terpenos e ácido salicílico.

Quando realizada a extração de seu óleo essencial este pode ser incorporado em diversas apresentações farmacêuticas de uso tópico (SOUSA, 1991). Os constituintes químicos da planta, em especial do óleo essencial estão concentrados nos canais secretores e glândulas multicelulares individuais situados na flor e no receptáculo, e estes são responsáveis pelo efeito fitoterápico (PEREIRA et al., 2001; PEREIRA et al., 2005; PEREIRA et al., 2008). A parte comercializada e com maior valor para a indústria são os capítulos florais secos (SOUSA, 1991).

A redução do teor de óleo essencial da camomila é inevitável quando submetida a diferentes temperaturas (BORSATO et al. , 2005a) e velocidades do ar (BORSATO et al., 2005b) de secagem. Alguns dos constituintes do óleo essencial da camomila podem ser recuperados durante processos de secagem à $70^{\circ} \mathrm{C}$ (BORSATO et al., 2007), à 60 C (BORSATO et al., 2008a) e à $80^{\circ} \mathrm{C}$ (BORSATO et al., 2008b) e suas propriedades 
físico-químicas foram simultaneamente caracterizadas por BORSATO et al. (2008c), indicando grande potencial para sua utilização em formulações farmacêuticas.

O termo alelopatia foi citado pela primeira vez em 1937 pelo alemão Hans Molish, citada por diversos autores ao longo dos anos até ser definida pela International Allelopathy Society como processos que envolvem a produção de metabólitos secundários por plantas e microrganismos que influenciam no crescimento e desenvolvimento de sistemas biológicos com efeitos positivos e negativos (MALHEIROS \& PEREZ, 2001; PINTO et al., 2002).

Visto que a produção anual desta espécie é significativa e que sua secagem gera uma quantidade de águas recuperadas também significativa, este trabalho teve como objetivo submeter as águas recuperadas a um teste alelopático para verificar o comportamento destas frente a sementes de Lactuca sativa e assim apresentar uma nova utilização às água recuperadas.

\section{MATERIAL E MÉTODOS}

Os solventes e reagentes utilizados apresentaram grau de pureza analítico (Merck). A espécie vegetal foi coletada entre os meses de outubro e dezembro de 2004 no município de Campo Largo, Paraná. Após a separação dos capítulos florais soltos, estes foram levados ao secador modelo Souza Cruz® com capacidade para cerca de uma tonelada de capítulos florais. Simultaneamente a secagem à $70^{\circ} \mathrm{C}$, obteve-se $\mathrm{o}$ condensado (água recuperada), límpido e de aroma agradável, a partir do vapor d'água que foi conduzido ao condensador. A cada três horas, durante o processo de secagem foi medido o volume deste condensado, o qual foi submetido ao processo de hidrodestilação para determinação do teor de óleo essencial. A identificação e quantificação dos componentes do óleo essencial foram realizadas conforme descrito em BORSATO et al. (2007).

Para o ensaio alelopático utilizaram-se sementes de Lactuca sativa (DIAS, 2005; DIAS et al., 2005) e as águas recuperadas obtidas em três, seis e nove horas de secagem de $C$. recutita. Para tratamento controle da germinação e do crescimento utilizou-se água destilada sob as mesmas condições. Para verificação da germinação, procedeu-se leitura diária durante sete dias com retirada das sementes consideradas germinadas conforme descrito por DE FEO et al. (2002) e ADEGAS et al. (2003), ou seja, quando se tornou visível a protrusão da radícula através do tegumento. A abertura diária das caixas Gerbox foi realizada em fluxo laminar. Calculou-se o índice da velocidade de germinação segundo MAGUIRE (1962).

Para verificação do crescimento realizou-se ao final do sétimo dia de experimento, leitura do crescimento do hipocótilo e da radícula em papel milimetrado. 
Os dados obtidos com a germinação e as leituras de crescimento do hipocótilo e da radícula foram submetidos ao Teste de Scott-Knott $(5 \%$ de probabilidade) (FERREIRA, 2000). O tratamento foi considerado efetivo quando todas as repetições estiveram no mesmo grupo de médias.

\section{RESULTADOS E DISCUSSÃO}

O hipocótilo e a radícula são originados a partir do eixo embrionário, parte vital da semente com tecido meristemático em duas extremidades, com condições de crescimento para dois sentidos, o das raízes (radícula) e o do caule (hipocótilo), originando plântula com condições de fixação ao solo e de fotossintetisar substâncias necessárias (CARVALHO \& NAKAGAWA, 1983). Nesta perspectiva, sabe-se que a influência em apenas um dos sentidos já é suficiente para originar plântulas anormais.

Os resultados do ensaio alelopático (Tabela 1) demonstram a capacidade das águas recuperadas de [Chamomilla recutita (L.) Rauschert] influenciarem a germinação e crescimento do hipocótilo de Lactuca sativa, independentemente do tempo de secagem.

TABELA 1 - RESULTADO DO ENSAIO ALELOPÁTICO EM ÁGUAS RECUPERADAS DE Chamomilla recutita APÓS SECAGEM À $70^{\circ} \mathrm{C}$

\begin{tabular}{|c|c|c|c|}
\hline \multicolumn{4}{|c|}{ INDICE DE VELOCIDADE DE GERMINAÇÃO (IVG) } \\
\hline $\operatorname{Ar} 3=6,1850 \mathrm{a}$ & $\operatorname{Ar} 6=5,6675 a$ & $\operatorname{Ar} 9=5,0450 a$ & Controle $=7,5425 \mathrm{~b}$ \\
\hline \multicolumn{4}{|c|}{ CRESCIMENTO DA RADÍCULA E DO HIPOCÓTILO } \\
\hline Radícula & \multicolumn{3}{|c|}{ Hipocótilo } \\
\hline $\operatorname{Ar3}=21,80 \mathrm{a}$ & $\operatorname{Ar} 9=25,00 \mathrm{a}$ & $\operatorname{Ar} 3=18,60 \mathrm{~b}$ & $\operatorname{Ar} 9=17,40 b$ \\
\hline $\operatorname{Ar} 3=19,20 a$ & $\operatorname{Ar} 9=26,70 \mathrm{a}$ & Ar3 $=14,80 b$ & $\operatorname{Ar} 9=17,10 b$ \\
\hline $\operatorname{Ar3}=20,80 a$ & $\operatorname{Ar} 9=26,90 \mathrm{a}$ & Ar3 $=18,80 b$ & $\operatorname{Ar} 9=19,20 b$ \\
\hline $\operatorname{Ar} 3=13,80 a$ & $\operatorname{Ar} 9=21,40 a$ & Ar3 $=13,00 \mathrm{~b}$ & $\operatorname{Ar} 9=18,40 b$ \\
\hline $\operatorname{Ar6}=25,80 a$ & Controle $=24,60 \mathrm{a}$ & Ar6 $=16,30 \mathrm{~b}$ & Controle $=36,40 \mathrm{a}$ \\
\hline $\operatorname{Ar6}=22,30 \mathrm{a}$ & Controle $=25,10 \mathrm{a}$ & $\operatorname{Ar} 6=16,60 \mathrm{~b}$ & Controle $=37,80 \mathrm{a}$ \\
\hline $\operatorname{Ar6}=17,40 a$ & Controle $=25,80 \mathrm{a}$ & $\operatorname{Ar} 6=12,40 \mathrm{~b}$ & Controle $=28,10 \mathrm{a}$ \\
\hline $\operatorname{Ar} 6=22,20 \mathrm{a}$ & Controle $=25,20 \mathrm{a}$ & $\operatorname{Ar} 6=18,30 \mathrm{~b}$ & Controle $=28,40 \mathrm{a}$ \\
\hline
\end{tabular}

NOTAS: (1) Ar3, Ar6 e Ar9 = água recuperada após 3, 6 e 9 horas, respectivamente. (2) Médias seguidas com a mesma letra na mesma linha para o IVG e nas colunas para o crescimento da radícula e do hipocótilo, não diferem estatisticamente entre si pelo Teste de Scott Knott $(p<0,05)$. 
Ao verificar os resultados das análises de CG-EM no óleo essencial extraído a partir de águas recuperadas durante o processo de secagem da camomila à $70^{\circ} \mathrm{C}$ observa-se que o tempo de secagem foi significativo somente para o alfa-pineno, ocorrendo diminuição de sua concentração até a sexta hora, enquanto que numericamente, observa-se aumento de cariofileno, artemísia cetona, 3-careno e outros e redução para as substâncias alfa-bisabolol, camazuleno e óxido de bisabolol (BORSATO et al., 2007). Portanto, com exceção do alfa-pineno, as concentrações dos constituintes do óleo essencial da camomila são alteradas apenas numericamente, sem apresentar comportamento peculiar em relação às condições de tempo de secagem (BORSATO et al., 2007).

\section{CONCLUSÃO}

Conclui-se que, apesar das águas recuperadas de [Chamomilla recutita (L.) Rauschert] após secagem à $70^{\circ} \mathrm{C}$ apresentarem algumas diferenças em sua composição química, estas não foram suficientes para alterar o potencial alelopático da espécie. Mais ensaios serão realizados, com o intuito de verificar a capacidade destas águas recuperadas em influenciar outros sistemas biológicos.

\section{REFERÊNCIAS}

ADEGAS, F.S.; VOLL, E.; PRETE, C.E.C. Embebição e germinação de sementes de picão-preto (Bidens pilosa). Planta Daninha, v.21, n.1., p.21-25, 2003.

BORSATO, A.V.; DONI-FILHO, L.; AHRENS, D.C. Secagem da camomila (Chamomilla recutita (L.) Rauschert) sob diferentes temperaturas do ar. Revista Brasileira de Plantas Medicinais, n.7, p. 77-85, 2005a.

BORSATO, A.V.; DONI-FILHO L.; AHRENS, D.C. Secagem da camomila (Chamomilla recutita (L.) Rauschert) sob diferentes vazões específicas do ar. Revista Brasileira de Plantas Medicinais, n.7, p.65-71, 2005b.

BORSATO, A.V.; DONI-FILHO L.; CÔCCO, L.C.; PAGLIA, E.C. Rendimento e composição do óleo essencial da camomila [Chamomilla recutita (L.) Rauschert] submetida à secagem à $70^{\circ} \mathrm{C}$. Semina: Ciências Agrárias, n.28, p. 635-644, 2007. 
BORSATO, A.V.; DONI-FILHO L.; CÔCCO, L.C.; PAGLIA, E.C. Rendimento e composição química do óleo essencial da camomila [Chamomilla recutita (L.) Rauschert] submetida à secagem à $60^{\circ} \mathrm{C}$. Revista Brasileira de Plantas Medicinais, n.10, p. 29-36, 2008a.

BORSATO, A.V.; DONI-FILHO L.; RAKOCEVIC, M.; CÔCCO, L.C.; PAGLIA, E.C. Chamomile essential oils extracted from flower heads and recovered water during drying process. Journal of Food Processing and Preservation (no prelo), 2008b.

BORSATO, A.V.; DONI-FILHO L.; MIGUEL, O.G.; PAGLIA, E.C. Propriedades físicoquímicas do óleo essencial de camomila submetida à secagem de camada fixa. Revista Brasileira de Plantas Medicinais, n. 10, p.24-30, 2008c.

CARVALHO, N.M.; NAKAGAWA, J. Sementes: ciência, tecnologia e produção. Campinas: Fundação Cargill, 1983.

CORREAA JUNIOR, C. Influência das adubações orgânicas e química na produção de camomila [Chamomilla recutita (L.) Rauschert] e o seu óleo essencial. Jaboticabal, 1994, Curitiba-PR, 102p. Dissertação de Mestrado em agronomia. Universidade do Estado de São Paulo.

CORREAA JUNIOR,C., ROSSI, L. Camomilla recutita (L.) Rauschert: características, aplicação e curiosidades. Revista Globo Rural. Disponível em: http://www. Revistagloborural.com.Br/ GloboRural . Acesso em 8/11/2007.

COSTA, A.F. Farmacognosia. Lisboa : Fundação Calouste Gulbenkion, 1987.

COSTA, M.A.; DONI FILHO, L. Aspectos do processo de produção agrícola na cultura da camomila [Chamomilla recutita (L.) Rauschert] no município de Mandirituba, Paraná. Visão Acadêmica, n.3, p.49-56, 2002.

DALLA COSTA, M.A.; MIGUEL, M.D. Camomila aspectos sanitários da colheita ao armazenamento, $1^{\text {a }}$ ed, Secretaria de Estado da Agricultura e Abastecimento do Paraná / UFPR / Prefeitura Municipal de Mandirituba, 2001, 36p.

DALLA COSTA, M. A. Processo de produção agrícola da cultura da camomila no município de Mandirituba, PR, 2001, Curitiba-PR, 63p. Tese de Doutorado em agronomia, UFPR. 
DE FEO, V.; DE SIMONE, F.; SENATORE, F. Potential allelochemicals from the essential oil of Ruta graveolens. Phytochemistry, v.61, n.5, p.573-578, 2002.

DIAS, J.F.G.; CÍRIO, G.M.; MIGUEL, M.D.; MIGUEL, O.G. Contribuição ao estudo alelopático de Maytenus ilicifolia Mart. ex Reiss., Celastraceae. Revista Brasileira de Farmacognosia, v.15, p.220-223, 2005.

DIAS, J.F.G. Estudo alelopático aplicado de Aster lanceolatus, Willd. Curitiba- PR, 114p. Dissertação de Mestrado em Ciências Farmacêuticas, Universidade Federal do Paraná.

FARMACOPÉIA Brasileira. 4. ed. São Paulo: Atheneu, 1988.

FERREIRA, D.F. Sistema de análise de variância de dados balanceados (SISVAR). Pacote computacional. Lavras: UFLA, 2000.

LORENZI, H.; MATOS, F.J.A. Plantas Medicinais no Brasil: Nativas e Exóticas. São Paulo: Instituto Plantarum de estudos da flora, 2002.

MAGUIRE, J.D. Speed of germination - aid in selection and evaluation for seedling emergence and vigor. Crop Science, v.2, n.2, p.176-177, 1962.

MALHEIROS, A .; PERES, M. T. L. P. Alelopatia: interações químicas entre espécies. In: YUNES, R.A.; CALIXTO, J.B. Plantas medicinais sob a ótica da química medicinal moderna. Chapecó: Argos, 2001. p. 503-523.

OLIVEIRA, R. Paraná mantém liderança na produção de camomila. Paraná Online. Disponível em: http://www.paraná-online.com.br. Acesso em 09/09/2009.

PEREIRA, N.P.; SATO, M.E.O.; MIGUEL, O.G.; PETRI, H.M.; RICETTI, L.M. Avaliação dos parâmetros de estabilidade, espalhabilidade e reológicos de emulsões tópicas formuladas com óleos vegetais usuais e com um novo óleo extraído da semente de camomila. Revista Portuguesa de Farmácia, v. LI, n.3, p.133-138, 2001.

PEREIRA, N.P; MIGUEL O.G; MIGUEL M.D. Composição química do óleo fixo obtido dos frutos secos da [Chamomilla recutita (L.) Rauschert] produzida no município de Mandirituba, PR. Revista Brasileira de Farmacognosia, v.15, n.4, p.334-337, 2005.

PEREIRA, N.P; MIGUEL O.G; MIGUEL M.D; CUNICO, M. Promising new oil derived 
from seeds of Chamomilla recutita (L.) Rauschert produced in southern Brazil. Journal Oil Chemists'Society, v.85, n.5, p.493-494, 2008.

PINTO, A.C.; SILVA, D.H.S.; BOLZANI, V.S.; LOPES, N.P.; EPIFANIO, R.A. Produtos naturais: atualidade, desafios e perspectivas. Química Nova, v.25, n.1, 2002. Disponível em :www.scielo.br. Acesso em: 22 abr. 2004.

SALAMON, I. Chamomile: a medicinal plant. The Herb, Spice and Medicinal Plant Digest, v.10, n.1, p. 1-4, 1992.

SOUSA, M.P.; MATOS, MEO.; MATOS, F.J.A.; MACHADO, M.IL.; CRAVEIRO, A.A. Plantas Medicinais Brasileiras. Fortaleza: Edições UFC, 1991.

TESKE, M.; TRENTINI, A.M. Compêndio de fitoterapia. Curitiba: Ed. Herbarium, 1995.

TUCKER, A.O.; DUKE, J.A.; FOSTER, S. Plantas aromáticas e medicinais: plantio, colheita, secagem, comercialização. São Paulo: Ícone, 1989. 